\title{
Electron spin resonance of thin films of organic light-emitting material tris(8-hydroxyquinoline) aluminum doped by magnesium
}

Donghyun Son ${ }^{\mathrm{a}}$, Kazuhiro Marumoto ${ }^{\mathrm{ab}, \mathrm{b},}$, Tokushi Kizuka ${ }^{\mathrm{a}}$, Yukihiro Shimoi ${ }^{\mathrm{c}, *}$

aDivision of Materials Science, University of Tsukuba, 1-1-1 Tennodai, Tsukuba, Ibaraki 305-8573, Japan

bapan Science and Technology Agency (JST), PRESTO, Kawaguchi, Saitama 322-0012, Japan

${ }^{\mathrm{c}}$ Nanosystem Research Institute (NRI), National Institute of Advanced Industrial Science and Technology (AIST), 1-1-1 Umezono, Tsukuba, Ibaraki 305-8568, Japan

E-mail: marumoto@ims.tsukuba.ac.jp; y.shimoi@aist.go.jp 


\begin{abstract}
We have successfully observed electron spin resonance (ESR) signals of radical anions in thin films of tris (8-hydroxyquinoline) aluminum ( $\left.\mathrm{Alq}_{3}\right)$, a compound widely used as electron transporting and luminescent layers in organic light-emitting diodes. To obtain definitely defined radical-anion states in $\mathrm{Alq}_{3}$, we doped $\mathrm{Alq}_{3}$ with $\mathrm{Mg}$ by co-evaporating these materials. The obtained $g$ value and peak-to-peak ESR linewidth $\Delta H_{\mathrm{pp}}$ of $\mathrm{Alq}_{3}$ radical anions are 2.0030 and $2.19 \mathrm{mT}$, respectively. Theoretical $g$ value and hyperfine interactions were calculated by density functional theory method, which are in good agreement with the experimental results. A quantitative evaluation of doping concentration was performed. We confirmed that doped charges are localized at deep trapping sites by the lineshape analysis and temperature dependence of the ESR signals. Morphological investigation using transmission electron microscopy clarified that the co-evaporated Mg atoms form clusters.
\end{abstract}

\title{
Keywords
}

Organic light emitting diodes (OLEDs); Tris(8-hydroxyquinoline) aluminum (Alq3); Electron spin resonance (ESR); Charge transfer; Charge-carrier state; Organic semiconductor 


\section{Introduction}

Since the first multilayered organic light-emitting diode (OLED) was introduced by Tang et al. [1], there have been drastic improvements in their device performance, and OLEDs are now ready to be commercialized [2,3]. OLEDs are currently ones of the strong candidates for next-generation displays and lightings owing to their excellent characteristics [2]. However, there are still several problems remain to be clarified such as degradation mechanisms and charge carrier states in OLEDs [3]. Investigating charge-carrier states in organic thin films is also a fundamental subject in basic science in addition to significant contribution for understanding device operation in OLEDs.

Charge carriers in organic materials can be described as radical ions and are usually accompanied with spins. Electron spin resonance (ESR) spectroscopy is suitable for investigating charge carriers in organic semiconductors and their devices from microscopic viewpoints, because it can directly observe electron spins. We have applied this spectroscopy not only to organic semiconducting materials [4] but also to organic semiconductor devices under operation such as organic field-effect transistors (OFETs) [5-7] and organic solar cells (OSCs) [8]. We also performed ESR study to investigate charge-carrier states of radical cations in $N, N^{\prime}$-Di(1-naphthyl)- $N, N^{\prime}$-diphenylbenzidine (NPB), a typical hole transporting material in OLEDs [9]. Pawlik et al. demonstrated that ESR study is also effective for OLEDs [10].

$\mathrm{Alq}_{3}$ is a compound widely used as a luminescent and electron transporting material in OLEDs [1]. The chemical structure of $\mathrm{Alq}_{3}$ is shown in Fig. 1. In OLED devices, electrons are injected from cathode to $\mathrm{Alq}_{3}$, which forms radical anions (negative polarons). Characterizing these radial anions is one of the most important issues associated with chargecarrier states of the real devices. There have been several reports for ESR characterization of 
$\mathrm{Alq}_{3}$ up to now [11-15]. However, these studies mainly observed residual paramagnetic species such as paramagnetic impurities or free radicals and did not identify the radical species in the ESR signals. Thus, a detailed ESR study on charge-carriers states in $\mathrm{Alq}_{3}$ has not yet been reported.

In this study, we investigate the charge-carrier states of $\mathrm{Alq}_{3}$ radical anions using the ESR method combined with density functional theory (DFT) calculation. To obtain definitely defined anion states of $\mathrm{Alq}_{3}$, doping by Mg was performed; such doping method is a common method to paramagnetize sample materials in the field of ESR study. Mg-doped $\mathrm{Alq}_{3}$ films are also used as an electron injecting layer whereby the dopants seem to lower a potential barrier at $\mathrm{Alq}_{3} / \mathrm{Al}$ interface $[16,17]$, and as an interconnecting layer in tandem-structure OLEDs [18]. In our ESR study, we clearly observed ESR signals due to $\mathrm{Alq}_{3}$ radical anions in Mg-doped $\mathrm{Alq}_{3}$ films. The observed $g$ value and ESR linewidth are well explained by theoretical results based on the DFT calculation. We also present the doping concentration of charge carriers by ESR intensity. Lineshape analysis and temperature dependence of the ESR signals indicate that observed spins are localized. Transmission electron microscopy (TEM) observation showed that doped Mg atoms form clusters.

\section{Experimental}

Mg-doped $\mathrm{Alq}_{3}$ film samples were prepared in a vacuum chamber under pressure of $2 \times 10^{-}$

${ }^{4} \mathrm{~Pa}$ by co-evaporating $\mathrm{Alq}_{3}$ and $\mathrm{Mg}$ on quartz substrates which were cleaned with isopropyl alcohol and acetone. Doping concentration was controlled by varying co-evaporation rate of $\mathrm{Alq}_{3}$ molecule and Mg atom. Subsequently, we deposited an additional Al layer on the Mgdoped $\mathrm{Alq}_{3}$ layer to protect Mg from oxidation. The thickness of layer for Mg-doped $\mathrm{Alq}_{3}$ and $\mathrm{Al}$ was 200 and $100 \mathrm{~nm}$, respectively. The area of the layer was $0.84 \mathrm{~cm}^{2}$. Each sample was 
then put in an ESR sample tube, which was evacuated down to $2 \times 10^{-5} \mathrm{~Pa}$, and then finally sealed under vacuum conditions or He gas atmosphere at $1.33 \times 10^{4} \mathrm{~Pa}$. ESR measurements on these samples were performed with a JEOL FA200 ESR spectrometer. The absolute values of the $g$ value, the ESR linewidth, and the number of spins of the samples were calibrated using a $\mathrm{Mn}^{2+}$ marker as a standard sample. The number of spins of the samples was obtained by integrating the ESR signal twice and by comparing with that of the $\mathrm{Mn}^{2+}$ marker in which the number of spins is known. TEM measurements were performed on $\mathrm{Alq}_{3}$ :Mg films that were deposited on a microgrid without Al layers.

DFT calculations were performed for an isolated $\mathrm{Alq}_{3}$ radical anion with the B3LYP functional and the 6-31G(d) basis set. The geometry is fully optimized for its meridional isomer. The calculations were carried out with the Gaussian 09 package [19].

\section{Results and Discussion}

By using Mg doping, we have successfully observed ESR spectra of $\mathrm{Alq}_{3}$ radical anions in Mg-doped $\mathrm{Alq}_{3}$ thin films. This indicates that charge transfer between $\mathrm{Mg}$ and $\mathrm{Alq}_{3}$ takes place. The observed ESR spectra are shown in Fig. 2 for the films deposited by coevaporating $\mathrm{Alq}_{3}$ and $\mathrm{Mg}$ in the ratio of 1:1 (solid curve) and 1:0.5 (dashed curve) for $\mathrm{Alq}_{3}$ molecules and $\mathrm{Mg}$ atoms. Signals around $316 \mathrm{mT}$ and $325 \mathrm{mT}$ are marker signals of a standard sample of $\mathrm{Mn}^{2+}$. $\mathrm{Alq}_{3}$ molecules are originally nonmagnetic, while magnetic moments arise when electrons are injected into $\mathrm{Alq}_{3}$ molecules [20]. Actually, our ESR measurement on a pure $\mathrm{Alq}_{3}$ thin film did not show any ESR signals, which means the amount of paramagnetic species in the undoped sample is under detection sensitivity. Our observation of the spectra in the doped samples is consistent with previous report that the $\mathrm{Alq}_{3} / \mathrm{Mg}$ interface forms an ohmic contact under vacuum condition and a large amount of 
charge carriers enter the $\mathrm{Alq}_{3}$ layer without applying voltage [21].

As seen in Fig. 2, both $g$ value (2.0030) and peak-to-peak ESR linewidth $\Delta H_{\mathrm{pp}}(2.19 \mathrm{mT})$ do not change with each other in spite of the different doping ratios. Both samples did not show any angular dependence to external magnetic field, reflecting amorphous nature of molecular orientation in thin films. The three principal $g$ values are calculated as 2.0024, 2.0029, and 2.0032 at the B3LYP/6-31G(d) level. These theoretical values are excellently consistent with the observed $g$ value. This consistency strongly supports our assignment of $\mathrm{Alq}_{3}$ radical anions in the ESR spectra, since the $g$ value is a fingerprint to identify the molecular spices in ESR measurements. The observed single ESR spectrum is reasonable, when we consider the $g$ shifts small enough compared with the linewidth in the samples of amorphous nature.

Next, we discuss the linewidth of the ESR spectra. The $\Delta H_{\mathrm{pp}}$ of $2.19 \mathrm{mT}$ of $\left(\mathrm{Alq}_{3}\right)^{-}$looks somewhat broad compared to other organic materials. For example, $\Delta H_{\mathrm{pp}}$ of $0.19 \mathrm{mT}$ and 0.01 $\mathrm{mT}$ was reported for hole carriers in pentacene and rubrene FETs, respectively [6,7]. An ESR linewidth reflects the motion of charge carriers and the magnitude of hyperfine interactions. For example, the ESR linewidths in pentacene and rubrene FETs are narrowed because of the mobile nature of carriers in addition to small hyperfine interactions. Here, we examine the hyperfine interaction in $\left(\mathrm{Alq}_{3}\right)^{-}$molecule. (We will discuss the effects of motion of charges later.) Table 1 summarizes calculated anisotropic hyper fine coupling constants for the atoms that have nuclear spins in natural abundance. The table also includes the charge and spin densities, $\rho$ and $s$, on each atom of an $\mathrm{Alq}_{3}$ anion evaluated by the natural population analysis. Figure 3 shows spin density profile calculated by the DFT method. Large spin density on an atom gives rise to a large hyperfine interaction. For a carbon atom, an electron interacts not with this carbon nucleus itself but with a hydrogen nucleus covalently bonded to it. As seen 
in Table 1, the calculated hyperfine interactions in $\left(\mathrm{Alq}_{3}\right)^{-}$show that one nitrogen atom (N2) and three hydrogen atoms (H7, H9, and H15) have absolute value of hyperfine interaction more than $0.5 \mathrm{mT}$. For atom numbering, see Fig. 1 . The hyperfine interaction with a nitrogen atom splits one resonance line into three, because the natural abundant ${ }^{14} \mathrm{~N}$ nucleus has nuclear spin $I=1$. This could make ESR linewidth further broader. For these reasons, we can expect an ESR signal with broad linewidth as we observed.

Next, we discuss the motion of charges doped in the films by means of lineshape analysis and the temperature dependence of the ESR signals. The shape of the ESR spectra was identified as Gaussian. This is evidence that the injected charges are deeply trapped and localized without motional narrowing. In order to further confirm the localization of charge carriers, temperature dependence of the $\Delta H_{\mathrm{pp}}$ was measured with the sample in the ratio of 1:1 in the temperature range between 4.2 and $300 \mathrm{~K}$. Generally, in case of highly mobile charge carriers, $\Delta H_{\mathrm{pp}}$ is expected to be narrowed as increasing the temperature because of motional narrowing. Our previous study on NPB thin films doped by iodine vapor showed that the $\Delta H_{\mathrm{pp}}$ is considerably narrowed as increasing the temperature due to the motional narrowing [9]. On the other hand, in this study, only small temperature dependence was observed, indicating almost no motional narrowing behavior for charges in Mg-doped $\mathrm{Alq}_{3}$. Thus, we confirm that the majority of the charge carriers are localized at deep trapping sites, implying that the binding energy of these trapping states are much larger than room temperature.

Here, we evaluate quantitatively the number of the charges transferred from $\mathrm{Mg}$ to $\mathrm{Alq}_{3}$. Assuming that the $\mathrm{Alq}_{3}$ molecules are singly charged, the number of charges with spins in the films is evaluated as $3.94 \times 10^{19}$ and $1.85 \times 10^{19} \mathrm{~cm}^{-3}$ for the samples in the ratio of $1: 1$ and 1:0.5, respectively. If we assume that charges are distributed uniformly over the film, we can 
evaluate the doping concentration in these films, that is, a percentage of charged molecules in total $\mathrm{Alq}_{3}$ molecules in the film. The later number was calculated from molecular weight, the volume in the films, and density reported in Ref. 22. The doping concentration was calculated to be $2.2 \%$ and $1.0 \%$ for the samples in the ratio of $1: 1$ and $1: 0.5$, respectively. In appearance, the doping concentration almost linearly increases with the co-evaporating ratio.

Next, we performed morphological investigation of the samples using TEM. Figure 4 shows a TEM image of the 1:0.5 sample. We can clearly observe that the clusters of Mg are formed. These Mg clusters were also found in the 1:1 sample. Therefore, we can confirm that Mg atoms in co-evaporated films tend to be condensed, rather than being dispersed in an atom level. This result probably indicates that the charge transfer takes place at the interface between $\mathrm{Alq}_{3}$ molecules and $\mathrm{Mg}$ clusters. The region specified by $\mathrm{Alq}_{3}: \mathrm{Mg}$ in the image is amorphous nature. We didn't confirm that $\mathrm{Mg}$ actually exists in the region because $\mathrm{Mg}$ clusters less than $0.5 \mathrm{~nm}$ cannot be detected by the TEM observation.

Finally, we comment on the energy level of $\mathrm{Alq}_{3}$. When the lowest unoccupied molecular orbital (LUMO) level of $\mathrm{Alq}_{3}(3.1 \mathrm{eV}$ ) [17] is compared with the work function of $\mathrm{Mg}$ (3.66 $\mathrm{eV}$ ), charge transfer seems to be hard to take place between $\mathrm{Alq}_{3}$ and $\mathrm{Mg}$. However, we also need to consider the relaxation of the molecular structure after accepting an electron as well as the Coulomb attraction between the $\mathrm{Alq}_{3}$ anion and cationic Mg cluster (or surface). Both of them stabilize the charge transfer state and may result in the formation of a gap state lower than the LUMO. A previous theoretical work pointed out the formation of gap state in the system of $\mathrm{Alq}_{3}$ with a Mg atom [23]. Thus, it is likely that charge transfer, at least in part, takes place.

\section{Conclusion}


We have successfully observed ESR signals of radical anion states of $\mathrm{Alq}_{3}$ and performed for the first time detailed investigation of radical anion states of $\mathrm{Alq}_{3}$ based on the ESR spectroscopy. In order to obtain definitely defined radical-anion states of $\mathrm{Alq}_{3}$, we coevaporated $\mathrm{Alq}_{3}$ with $\mathrm{Mg}$ to form deposited thin films. The experimental ESR parameters of the $g$ values and ESR linewidth are well explained by theoretical results using the DFT method. The lineshape and temperature dependence of the obtained ESR spectra show localized nature of charges. The number of charges with spins, in appearance, increases proportionally to the ratio of $\mathrm{Mg}$ atoms in the co-evaporation. The TEM observation showed that Mg clusters are formed in these samples. Based on this study, we can expect that the ESR spectroscopy is useful to study charge states in OLEDs and offers a basis to device characterization.

\section{Acknowledgements}

The authors would like to thank Prof. Y. Furukawa for his valuable comments on fabrication of the sample. This work was partly supported by Grants-in-Aid for Scientific Research (No. 24560004 and No. 22340080) from the Japan Society for the Promotion of Science (JSPS) and by JST, PRESTO. 


\section{Table captions}

Table 1. Charge and spin densities, $\rho$ and $s$, on the Alq3 atoms in its anion state evaluated by the natural population analysis at the B3LYP/6-31G(d) level. The anisotropic hyper fine coupling (hfc) constants are also included for the Al, $\mathrm{N}$, and $\mathrm{H}$ atoms that have nuclear spins in natural abundance. See Fig. 1 for the atom numbering.

\section{Figure captions}

Figure 1. Chemical structure and atomic numbering of $\mathrm{Alq}_{3}$.

Figure 2. ESR spectra of Mg-doped $\mathrm{Alq}_{3}$ thin films. Solid and dashed lines show the ESR spectrum of the sample co-evaporated in the ratio of 1:1 and 1:0.5 for $\mathrm{Alq}_{3}$ molecule and $\mathrm{Mg}$ atom, respectively.

Figure 3. Spin density distribution profile of an $\left(\mathrm{Alq}_{3}\right)^{-}$calculated at the B3LYP/6-31G(d) level.

Figure 4. TEM image of the Mg-doped $\mathrm{Alq}_{3}$ film co-evaporated on a microgrid. The molecular ratio, $\mathrm{Alq}_{3}: \mathrm{Mg}$, of the film is 1:0.5. 


\section{References}

[1] C.W. Tang, S.a. VanSlyke, Appl. Phys. Lett. 51 (1987) 913.

[2] H. Sasabe, J. Kido, Chem. Mater. 23 (2011) 621.

[3] F. So, D. Kondakov, Adv. Mater. 22 (2010) 3762.

[4] K. Marumoto, Y. Muramatsu, Y. Nagano, T. Iwata, S. Ukai, H. Ito, S. Kuroda, Y. Shimoi, S. Abe, J. Phys. Soc. Jpn. 74 (2005) 3066.

[5] K. Marumoto, S. Kuroda, T. Takenobu, Y. Iwasa, Phys. Rev. Lett. 97 (2006) 256603.

[6] K. Marumoto, M. Kato, H. Kondo, S. Kuroda, N. C. Greenham, R. H. Friend, Y. Shimoi, S. Abe, Phys. Rev. B 79 (2009) 245204.

[7] K. Marumoto, N. Arai, H. Goto, M. Kijima, K. Murakami, Y. Tominari, J. Takeya, Y. Shimoi, H. Tanaka, S. Kuroda, T. Kaji, T. Nishikawa, T. Takenobu, Y. Iwasa, Phys. Rev. B 83 (2011) 075302.

[8] K. Marumoto, T. Fujimori, M. Ito, T. Mori, Adv. Energy Mater. 2 (2012) 591.

[9] K. Azuma, D. Son, K. Marumoto, M. Kijima, Y. Shimoi, Chem. Lett. 41 (2012) 191.

[10] T.D. Pawlik, D.Y. Kondakov, W.J. Begley, R.H. Young, J. Soc. Inf. Display 18 (2010) 277.

[11] M.N. Grecu, A Mirea, C. Ghica, M. Cölle, M. Schwoerer, J. Phys.: Condens. Matter 17 (2005) 6271.

[12] B. Kanchibotla, S. Pramanik, S. Bandyopadhyay, M. Cahay, Phys. Rev. B 78 (2008) 193306.

[13] C.Y. Kwong, A.B. Djurišić, W.C.H. Choy, D. Li, M.H. Xie, W.K. Chan, K.W. Cheah, P.T. Lai, P.C. Chui, Mater. Sci. Eng. B 116 (2005) 75.

[14] V.A.L. Roy, R.B. Pode, T.K.G. Rao, A.B. Djurišić, G. Baldacchini, Mater. Sci. Eng. B 106 (2004) 85.

[15] V.A.L. Roy, C.Y. Kwong, A.B. Djurišić , R.B. Oide, T.J. Gundu Rao, W.K. Chan, Int. Soc. Opt. Engineer. Proc. 5214 (2004) 283.

[16] K. Hong, S.Y. Kim, W.K. Kim, J.L. Lee, Electrochem. Solid-State Lett. 10 (2007) H85.

[17] C.H. Lin, C.T. Chou, Y.H. Chen, M.H. Wu, J.H. Lee et al., J. Appl. Phys. 109 (2011) 083541. 
[18] C.W. Law, K.M. Lau, M.K. Fung, M.Y. Chan, F.L. Wong, C.S. Lee, S.T. Lee, Appl. Phys. Lett. 89 (2006) 133511.

[19] M.J. Frisch et al., Gaussian 09 (Revision A.02), Gaussian, Inc., Wallingford CT, (2009).

[20] K. Tarafder, B. Sanyal, P.M. Oppeneer, Phys. Rev. B 82 (2010) 060413.

[21] M. Kiy, I. Biaggio, M. Koehler, P. Günter, Appl. Phys. Lett. 80 (2002) 4366.

[22] M. Brinkmann, G. Gadret, M. Muccini, C. Taliani, N. Masciocchi, A. Sironi, J. Am. Chem. Soc. 122 (2000) 5147.

[23] R.Q. Zhang, X.Y. Hou, S.T. Lee, Appl. Phys. Lett. 74 (1999) 1612. 


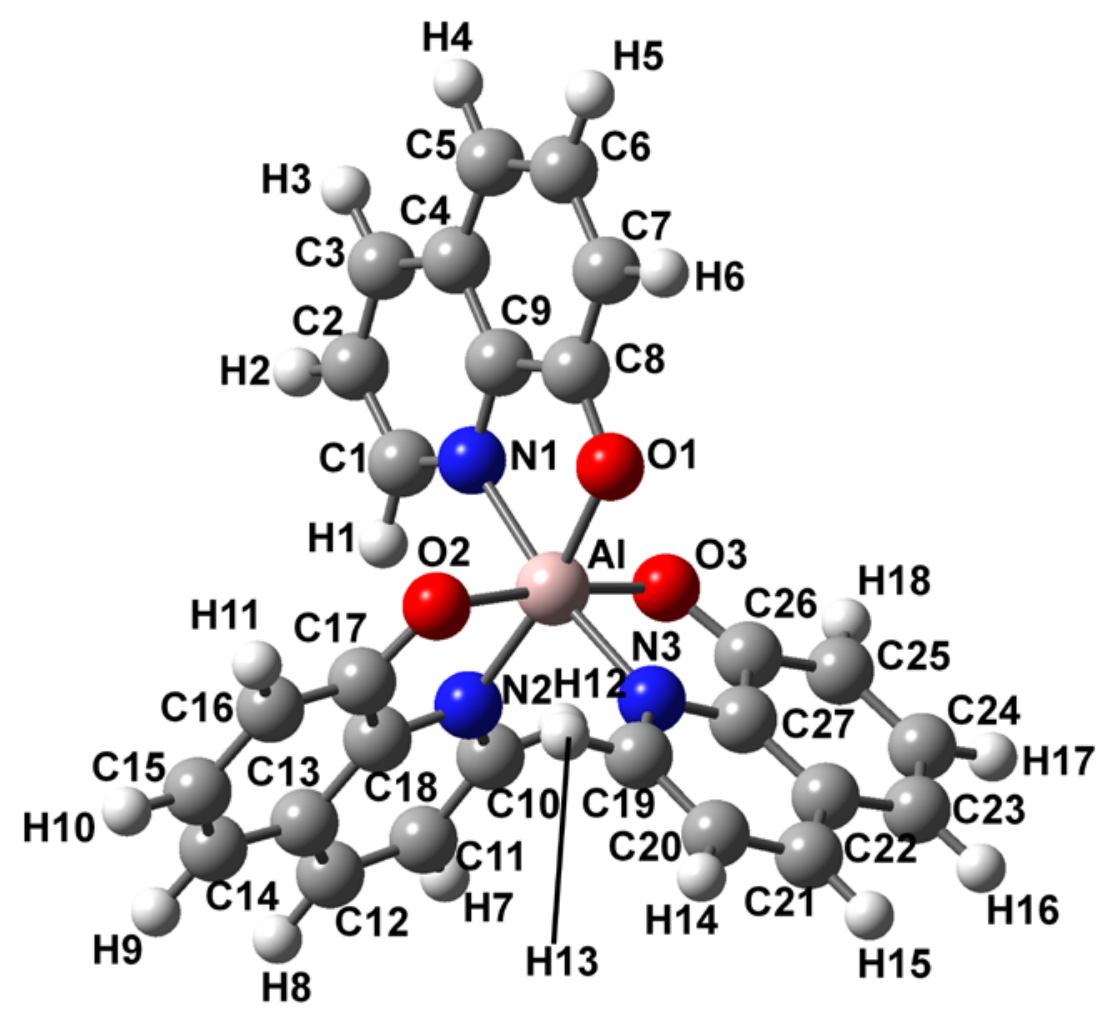

Figure 1. D. Son et al. 


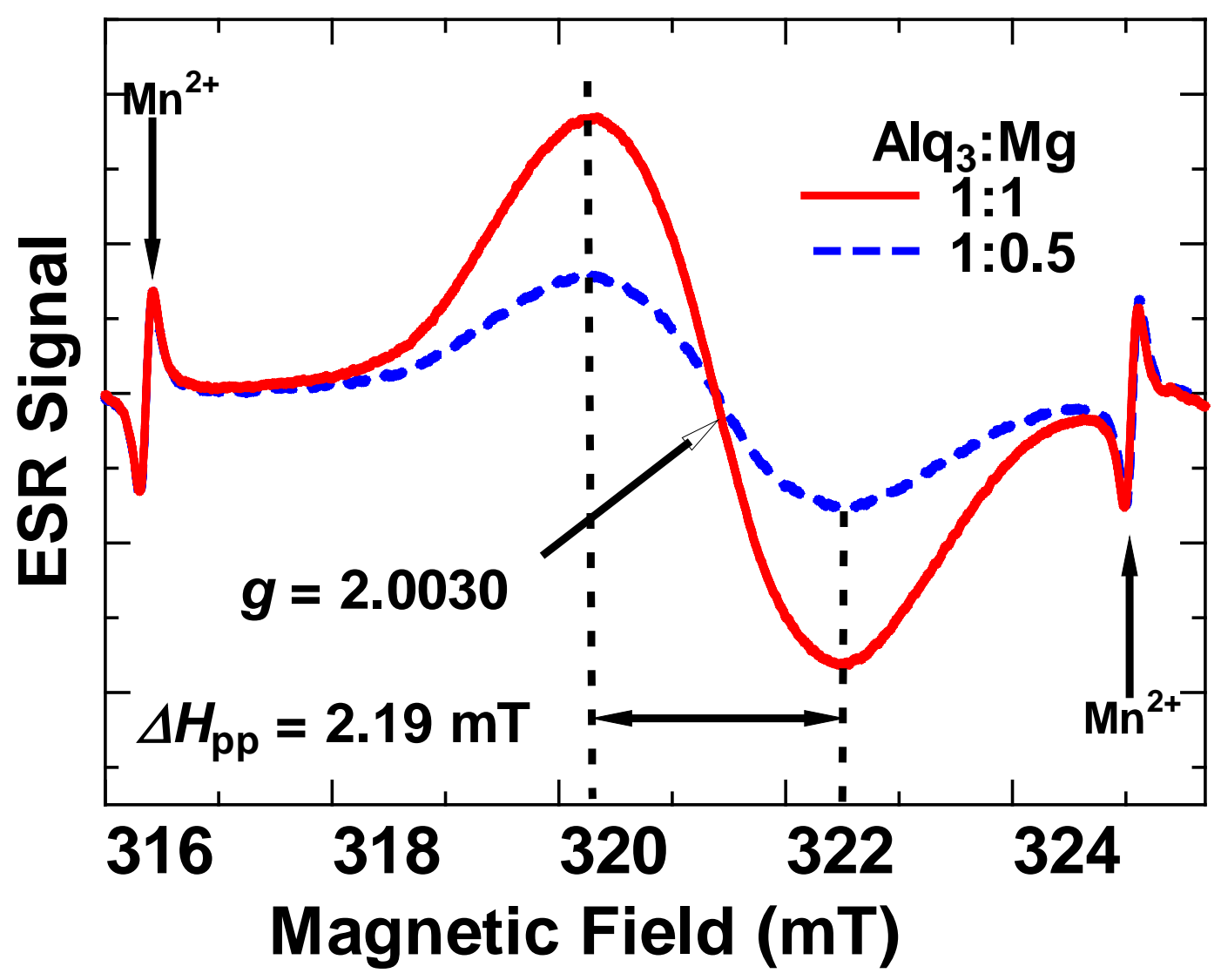

Figure 2. D. Son et al. 


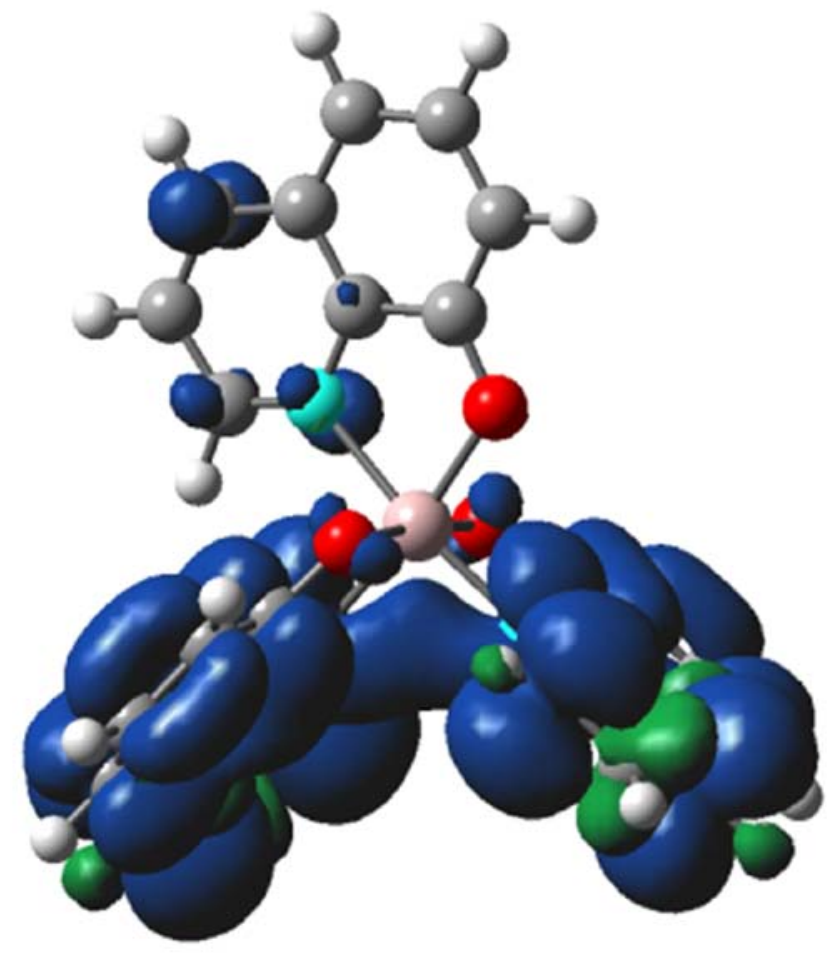

- Al

- N

- 0

- c

$\mathrm{H}$

Figure 3. D. Son et al. 


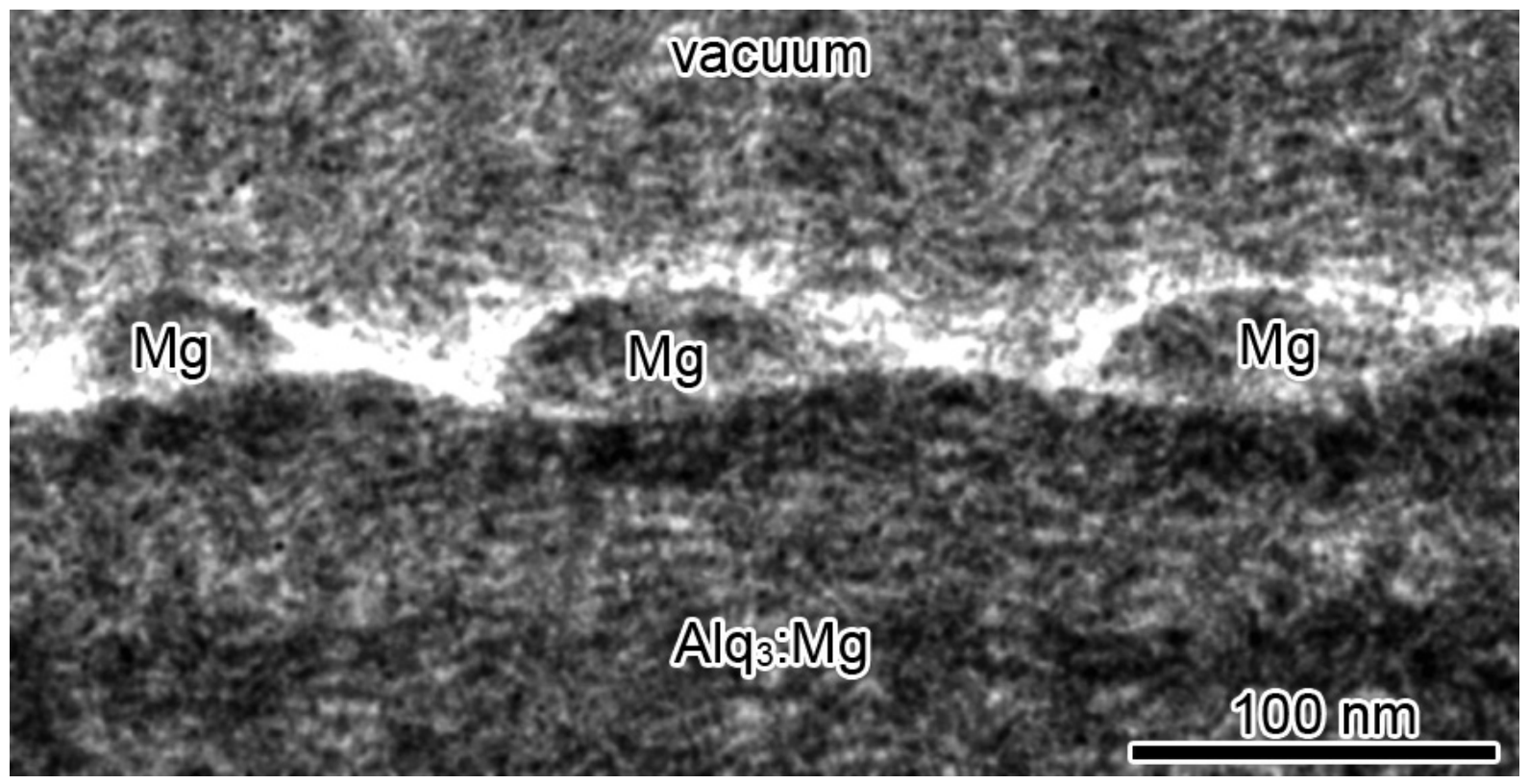

Figure 4. D. Son et al. 


\begin{tabular}{|c|c|c|c|c|c|}
\hline Atom & $\rho$ & $S$ & & $\mathrm{C} \quad[\mathrm{mT}]$ & \\
\hline $\mathrm{Al}$ & 1.981 & 0.001 & -0.189 & -0.172 & -0.116 \\
\hline N1 & -0.532 & 0.004 & 0.047 & 0.053 & 0.095 \\
\hline O1 & -0.791 & 0.001 & & & \\
\hline C1 & 0.055 & 0.002 & & & \\
\hline C2 & -0.286 & -0.000 & & & \\
\hline C3 & -0.178 & 0.007 & & & \\
\hline C4 & -0.081 & -0.001 & & & \\
\hline C5 & -0.297 & 0.001 & & & \\
\hline C6 & -0.212 & 0.000 & & & \\
\hline C7 & -0.324 & 0.000 & & & \\
\hline C8 & 0.398 & 0.001 & & & \\
\hline C9 & 0.147 & 0.002 & & & \\
\hline H1 & 0.240 & -0.000 & -0.059 & -0.044 & 0.066 \\
\hline $\mathrm{H} 2$ & 0.235 & 0.000 & -0.014 & -0.008 & 0.019 \\
\hline H3 & 0.228 & -0.000 & -0.067 & -0.048 & -0.011 \\
\hline H4 & 0.219 & -0.000 & -0.010 & -0.009 & 0.007 \\
\hline H5 & 0.222 & -0.000 & -0.006 & -0.006 & 0.008 \\
\hline H6 & 0.234 & -0.000 & -0.010 & -0.006 & 0.012 \\
\hline N2 & -0.673 & 0.068 & 0.057 & 0.064 & 0.739 \\
\hline $\mathrm{O} 2$ & -0.849 & 0.002 & & & \\
\hline C10 & -0.013 & 0.071 & & & \\
\hline C11 & -0.297 & -0.018 & & & \\
\hline C12 & -0.257 & 0.133 & & & \\
\hline C13 & -0.080 & -0.015 & & & \\
\hline C14 & -0.296 & 0.022 & & & \\
\hline C15 & -0.237 & 0.007 & & & \\
\hline C16 & -0.327 & 0.008 & & & \\
\hline C17 & 0.347 & 0.016 & & & \\
\hline C18 & 0.129 & 0.013 & & & \\
\hline H7 & 0.232 & -0.002 & -0.666 & -0.496 & -0.201 \\
\hline H8 & 0.223 & 0.000 & -0.010 & 0.070 & 0.119 \\
\hline H9 & 0.214 & -0.004 & -1.152 & -0.781 & -0.336 \\
\hline H10 & 0.212 & -0.001 & -0.171 & -0.165 & -0.039 \\
\hline H11 & 0.214 & -0.000 & -0.093 & -0.080 & 0.009 \\
\hline H12 & 0.225 & -0.000 & -0.093 & -0.080 & -0.001 \\
\hline
\end{tabular}




\begin{tabular}{lrrrrr} 
N3 & -0.620 & 0.040 & 0.045 & 0.054 & 0.453 \\
O3 & -0.837 & 0.002 & & & \\
C19 & 0.026 & 0.045 & & & \\
C20 & -0.291 & -0.011 & & & \\
C21 & -0.225 & 0.083 & & & \\
C22 & -0.079 & -0.010 & & & \\
C23 & -0.294 & 0.014 & & & \\
C24 & -0.228 & 0.004 & & & \\
C25 & -0.327 & 0.004 & & & \\
C26 & 0.364 & 0.010 & & & \\
C27 & 0.140 & 0.009 & & & \\
H13 & 0.238 & -0.001 & -0.418 & -0.306 & -0.136 \\
H14 & 0.227 & 0.000 & -0.006 & 0.040 & 0.081 \\
H15 & 0.219 & -0.003 & -0.731 & -0.497 & -0.213 \\
H16 & 0.215 & -0.000 & -0.108 & -0.103 & -0.020 \\
H17 & 0.217 & -0.000 & -0.059 & -0.050 & 0.009 \\
H18 & 0.227 & -0.000 & -0.059 & -0.041 & -0.001 \\
\hline
\end{tabular}

Table 1. D. Son et al. 